British Journal of Nutrition (2021), 126, 1076-1090

doi:10.1017/S0007114520005000

(C) The Author(s), 2020. Published by Cambridge University Press on behalf of The Nutrition Society. This is an Open Access article, distributed under the terms of the Creative Commons Attribution licence (http://creativecommons.org/licenses/by/4.0/), which permits unrestricted re-use, distribution, and reproduction in any medium, provided the original work is properly cited.

\title{
Nature of the evidence base and frameworks underpinning dietary recommendations for prevention of non-communicable diseases: a position paper from the Academy of Nutrition Sciences
}

\author{
Christine M. Williams ${ }^{1 *}$, Margaret Ashwell ${ }^{1}$, Ann Prentice ${ }^{2,5}$, Mary Hickson ${ }^{3,6}$ and Sara Stanner ${ }^{4}$ on behalf of \\ the Academy of Nutrition Sciences ${ }^{1} \dagger$ \\ ${ }^{1}$ Trustees, Academy of Nutrition Sciences, 10 Cambridge Court, 210 Shepherds Bush Road, London W6 7NJ, UK \\ ${ }^{2}$ The Nutrition Society, London W6 7NJ, UK \\ ${ }^{3}$ British Dietetic Association, Birmingham B3 2TA, UK \\ ${ }^{4}$ British Nutrition Foundation, London WC1X 8TA, UK \\ ${ }^{5}$ MRC Nutrition and Bone Health Group, Cambridge CB2 OAH, UK \\ ${ }^{6}$ School of Health Professions, University of Plymouth, Plymouth PL6 8BH, UK \\ (Submitted 28 August 2020 - Final revision received 26 November 2020 - Accepted 27 November 2020 - First published online 10 December 2020 )
}

Abstract

This Position Paper from the Academy of Nutrition Sciences is the first in a series which describe the nature of the scientific evidence and frameworks that underpin nutrition recommendations for health. This first paper focuses on evidence which underpins dietary recommendations for prevention of non-communicable diseases. It considers methodological advances made in nutritional epidemiology and frameworks used by expert groups to support objective, rigorous and transparent translation of the evidence into dietary recommendations. The flexibility of these processes allows updating of recommendations as new evidence becomes available. For CVD and some cancers, the paper has highlighted the long-term consistency of a number of recommendations. The innate challenges in this complex area of science include those relating to dietary assessment, misreporting and the confounding of dietary associations due to changes in exposures over time. A large body of experimental data is available that has the potential to support epidemiological findings, but many of the studies have not been designed to allow their extrapolation to dietary recommendations for humans. Systematic criteria that would allow objective selection of these data based on rigour and relevance to human nutrition would significantly add to the translational value of this area of nutrition science. The Academy makes three recommendations: (i) the development of methodologies and criteria for selection of relevant experimental data, (ii) further development of innovative approaches for measuring human dietary intake and reducing confounding in long-term cohort studies and (iii) retention of national nutrition surveillance programmes needed for extrapolating global research findings to UK populations.

Key words: Nutrition: Evidence: Frameworks: Challenges

Food, nutrition and health have become a distinctive and increasingly popular focus for many people. The Academy of Nutrition Sciences, formed in 2019, welcomes this widespread interest in the relationship between food and health and the greater scrutiny of the scientific evidence underpinning nutrition advice to the population. It recognises the complex nature of the evidence base and the need for better understanding of how that evidence is selected and brought together to provide advice on diet and health in different settings. This position paper from the
Academy is the first in a series of three papers which describe the nature of the scientific evidence and the processes that underpin diet and nutrition advice to populations for prevention of noncommunicable diseases, support health claims for specific foods and provide advice to patients with acute and chronic ill health.

This first paper focuses explicitly on the evidence which underpins dietary recommendations for prevention of noncommunicable diseases with a particular focus on CVD and cancers. The report considers the major advances made in

Abbreviations: RCTs, randomised controlled trials; RR, relative risk; SACN, Scientific Advisory Committee on Nutrition; WCRF, World Cancer Research Fund

* Corresponding author: Christine Williams, email c.m.williams@reading.ac.uk

$\dagger$ The Academy of Nutrition Sciences is an association of four member organisations including the Nutrition Society, Association for Nutrition, British Dietetic Association and British Nutrition Foundation, representing professionals involved in nutrition research, communication and application of research, nutrition education and professional regulation. 
nutritional epidemiology over the past 50 years. It outlines the key processes used by expert groups in developing recommendations for population dietary changes aimed at reducing risk of non-communicable diseases. It identifies some of the remaining challenges in this complex area of science and the need for more audience-specific communications on the nature of the evidence base in nutrition. Although this paper's focus is on the evaluation of effects of diets, foods and nutrients on non-communicable diseases, the Academy recognises the complex interplay between excess energy intakes, low physical activity levels and body weight gain as risk factors for many non-communicable diseases. Distinguishing these as three independent causal risk factors or as co-conspirators in disease causation remains a key challenge for those involved in public health research, practice and policy.

This report has not undertaken a systematic review of the different working practices, quality assessments or grading criteria used by the many authorities responsible for setting dietary recommendations worldwide. Some of the recommendations made in this position paper (see the 'Conclusions and recommendations' section) are focused on UK diet and policy issues; however, the analysis and findings are generalisable to many countries worldwide. We have included consideration of wellestablished processes and guidelines used by bodies outside the UK including the USA and European Union and those of the World Cancer Research Fund (WCRF) International. The literature reflects the international nature of the evidence base for diet and non-communicable disease including recent reviews which address particular areas of uncertainty, opportunity and challenge ${ }^{(1-3)}$.

\section{Epidemiology and the evolution of diet-disease hypotheses}

In the early post-war period, epidemiology was beginning to be used as a tool for studying relationships between environmental exposures and prevalence of certain diseases, for example, smoking and lung cancer. Some early epidemiological findings, such as between-country mortality data and changes in disease incidence within migrant groups, also began to report potential links between diet and incidence of non-communicable diseases such as $\mathrm{CVD}^{(4)}$. Concerns regarding the rising incidence of CVD in developed countries led to the setting up of case-control studies, which compared the diets of cases (patients with disease) from non-disease groups. These tended to support evidence for involvement of diet as a factor in the aetiology of CVD. Cohort studies began to measure baseline diets in order to follow incidence and mortality rates in groups whose diets had been measured prior to the onset of disease ${ }^{(4)}$. Unlike case-control studies, where dietary recall may be biased due to the impact of diagnosis, cohort studies had the advantage of measuring diet prior to the development of the disease of interest, as well as being able to evaluate diet associations for a wide range of disease outcomes. These early studies, now in their fifth or sixth decade of follow-up, have revealed valuable information on associations between diet and lifestyle risk factors for diseases such as CVD, cancers, type 2 diabetes and, increasingly, cognitive health. Cohort studies thus provided the basis for development of credible hypotheses concerning the role of Western diets in the aetiology of CVD and which were supported by plausible mechanisms, for example, via adverse effects on blood pressure and circulating cholesterol levels.

\section{Development of dietary recommendations for prevention of chronic disease}

These early observations prompted some countries and agencies to set up expert committees to make recommendations for reducing adverse effects of diet on health ${ }^{(5-8)}$. At the time, much of the evidence base comprised relatively small numbers of findings from observational studies (between-country, migrant groups, case-control and cohort studies), randomised controlled trials (RCTs) (where available) and animal feeding studies.

Although tentative dietary recommendations were made by the expert groups, the evidence was considered insufficient to support causal relationships between exposure to specific diets, foods or nutrients and risk of non-communicable diseases. The value of prospective cohort studies was recognised, but there were few of these at the time and their observational nature limited conclusions about causality. This emphasis on the causal nature of diet-diseases associations recognised that major changes in population diets would require a high level of certainty that these could yield reductions in disease incidence and reduced mortality. As experience grew it became clear that RCTs, considered the 'gold standard' for investigating causal effects of clinical interventions, were less suited to studying relationships between diet and non-communicable diseases over the decades of their aetiological pathways. Drawbacks identified from RCTs of diet and clinical outcomes include ${ }^{(3)}$ inability to fully control the dietary exposure of interest; challenges of identifying suitable substitutions for the food or nutrient of interest in control diets; limited duration of intervention (usually a few years) relative to the long time-course for the development of non-communicable diseases; high dropout and low compliance rates; risk of selection and reporting bias and limited generalisability of findings, particularly when conducted under atypical conditions (e.g. residential homes, hospitals, prisons) and within non-representative groups of the population.

Investigation of the impact of diet on CVD was aided by the availability of a robust risk biomarker, serum cholesterol. Many RCTs have since been conducted using serum cholesterol, an intermediate risk marker for CVD, as the primary outcome. Unlike RCTs of clinical outcomes, these can be conducted over weeks or a few months which makes them feasible to conduct on large numbers of participants while achieving good dietary compliance. These studies have formed an important part of the evidence base for effects of dietary fats on CVD risk. Glycated $\mathrm{Hb}$ has also been successfully used as a risk marker in preventative dietary strategies for type 2 diabetes. For some diseases, such as many cancers and dementias, no widely accepted markers are yet available, although this remains an active area of research.

\section{Assigning causality from observational epidemiology}

Increasing interest in the role of epidemiology in the study of disease causation led to much debate about assigning causal 
Table 1. Bradford Hill characteristics for causality: principles used in establishing epidemiological evidence for a causal relationship

\begin{tabular}{|c|c|}
\hline Characteristics & Description \\
\hline Temporality & $\begin{array}{l}\text { Temporal relation between presence of a } \\
\text { factor and occurrence of disease }\end{array}$ \\
\hline Strength of association & $\begin{array}{l}\text { Magnitude of the relative risk associated } \\
\text { between disease outcome and the } \\
\text { exposure of interest }\end{array}$ \\
\hline Consistency & $\begin{array}{l}\text { Extent to which findings are similar across the } \\
\text { body of the evidence }\end{array}$ \\
\hline $\begin{array}{l}\text { Biologic gradient } \\
\text { (dose-response) }\end{array}$ & $\begin{array}{l}\text { Observed relation between an exposure and } \\
\text { disease is related by the amount of } \\
\text { exposure of that factor for the disease }\end{array}$ \\
\hline Biological plausibility & $\begin{array}{l}\text { Knowledge of a mechanism of action for the } \\
\text { disease by a known factor }\end{array}$ \\
\hline Specificity & $\begin{array}{l}\text { Extent to which a single, well-characterised } \\
\text { factor can be consistently shown to be } \\
\text { present for a disease }\end{array}$ \\
\hline Analogy & $\begin{array}{l}\text { Causal interpretation is based on similarity of } \\
\text { an association with another association }\end{array}$ \\
\hline
\end{tabular}

relationships from observational studies. In the mid-1960s, Bradford Hill put forward a list of characteristics that might be used to assess strength of the epidemiological data for causal relationships ${ }^{(9)}$. These characteristics included strength (size) of associations, dose-response and consistency between different studies, which could be addressed in individual cohort studies (Table 1).

As larger studies of better design became available, use of the Bradford Hill framework supported further interrogation of the data for possible causal relationships. Assessment of biological plausibility was not considered amenable to interpretation from observational studies. Bradford Hill considered this an ongoing challenge saying: 'What is biologically plausible depends upon the biological knowledge of the day'. Modified versions of the Bradford Hill criteria and their underlying concepts continue to be widely used in assessing evidence from epidemiology, including evidence for diet-disease relationships. However, their precise wording, limitations and applicability continue to be debated.

\section{Advances in nutritional epidemiology - translation to dietary recommendations}

Since these early days of nutritional epidemiology, there has been significant expansion in the number of large-scale, highquality cohort studies, some of which have follow-up periods of 20-50 years. This allows for accumulation of detailed databases with larger numbers of disease cases and greater statistical power. Nevertheless some of the earliest studies used methodologies and tools for data and dietary collection and analysis which were not as advanced as some of the more recently established cohorts which may contribute to between-study heterogeneity in summated data.

Advances in nutritional epidemiology through new methodologies, skills and training and facilitated through open collaborative networks have allowed improvements in the quality and rigour of the epidemiological evidence base. Globally, the development of centres of excellence has helped set quality standards for research and rigorous peer review of research findings. Innovations in the conduct of cohort studies over the past 20-50 years include the developments in use of statistics that can reduce the impact of confounding between nutrients and/ or food categories; very large cohorts and long-term follow-up provide increased statistical power and allow sub-group analyses; improvements in dietary questionnaire design, data collection and more complete food and nutrient databases to reduce measurement error; repeat measurements of diet at intervals allowing for changes in diet over time to be measured. In recent years, there has also been increasing emphasis on dietary pattern analysis to understand holistic effects of whole foods and diets rather than specific nutrients. This reflects an increasingly accepted view that food and meal patterns provide a better foundation for developing food-based dietary guidelines, as well as a stronger basis for communicating advice to the general population ${ }^{(2)}$.

The development of underpinning methodologies for carrying out systematic reviews and meta- and pooled-analyses ${ }^{(10-13)}$ has greatly facilitated the interpretation of large, complex data sets. The steps involved in performing systematic reviews in the area of diet and health and some of the challenges specific to nutrition have previously been well described ${ }^{(10)}$. Systematic reviews are now standard methodologies used by expert panels, which enable the totality of the primary literature to be reviewed (see the 'Processes and frameworks used to structure assessment of the evidence underpinning dietary recommendations' section). There is less focus on narrative accounts of individual studies, with reduced risk of publication bias that can arise from over-reliance on highly cited reports. Summation of data via meta- and pooled-analysis allows the causal characteristics of effect size, dose-response, consistency, specificity and temporality to be addressed, assuming potential impacts of confounding and bias can be minimised, in order to judge the likelihood that any observed association might be causal.

Despite these improvements, the challenge of translating the evidence into dietary recommendations for whole populations remains a significant one. To support translation of the evidence into dietary recommendations, most panels carry out their work using standardised frameworks and grading systems which aid consistency in assessing strength of the evidence for causal links between diet and non-communicable diseases (see the 'Processes and frameworks used to structure assessment of the evidence underpinning dietary recommendations' section). Some panels apply their own grading systems ${ }^{(14)}$, others use their own in combination with published systems such as AMSTAR 2 and GRADE ${ }^{(15,16)}$. Grading systems which take account of particular considerations for assessing strength of the evidence related to diet and health are available but do not appear to have been cited in reports from expert groups as yet ${ }^{(17,18)}$.

Published reports from expert groups provide details of how they have applied these frameworks and grading criteria in drawing up their final recommendations. As well as supporting consistency in decision-making, grading criteria can help identify significant gaps or weaknesses in the evidence and allow clear recommendations for future research to be made. 
Notwithstanding the considerable advances that have been made in systematising approaches to the evidence, expert panels continue to debate many scientific and technical matters as they seek to reach consensus on their final recommendations. Many systematic reviews have been published that have drawn upon essentially the same data but come to different conclusions. Expert panels need to interrogate the reasons underlying these differences and use statistical expertise to judge some of the advanced statistical techniques used in these reviews. Their judgements need to take account of not only the quality of the evidence itself, which usually draws on an international database of studies, but also the applicability of the findings to the country in which the panel resides. These judgements can encompass a wide range of issues, but examples include the cultural relevance of the findings, prevalence of other health concerns and the susceptibility of specific sub-groups within the population.

\section{Experimental studies - biological plausibility and causality}

The Bradford Hill framework includes availability of evidence to support biological plausibility. This requires approaches outside epidemiology, although underlying mechanisms may be inferred from cohort studies when supported by available biomarker, body composition and genetic data (e.g. for use in Mendelian randomisation).

There is a substantial body of evidence concerning mechanisms which might underlie the relationships between diet and non-communicable diseases. Such studies, often referred to as experimental, mechanistic or biological, investigate the molecular, cellular, physiological and pathophysiological mechanisms that might be relevant to the development of specific non-communicable diseases, and the ability of putative mechanisms to be modified by specific diets, foods or nutrients. They can include in vitro, in vivo and ex vivo studies of the effects of relevant diets, foods, nutrients or other dietary components, ideally conducted under randomised conditions. These studies may involve live animal models, to test effects of diet and nutrition at various stages of disease process (e.g. cancers at many sites, CVD), or humans including healthy volunteers, those with pre-disease states or patients with disease. Both animal and human models can control factors other than the dietary exposure of interest, thereby avoiding confounding of the findings.

Animal models offer the advantage of lower variability and greater statistical power, due to common genetic background and the ability to sample using invasive procedures. Due to differences in key aspects of genetics, physiology, metabolism or toxicology, some animals do not provide suitable models for human diseases. Although compliance to experimental diets can be more readily assured and measured accurately in animal models, the literature includes many examples where extreme diets or high levels of supplements are fed, which are unfeasible or potentially harmful in human populations. Although such studies may provide useful insight to putative mechanisms, their extrapolation to the human diet situation is not appropriate and so of limited value in drawing up dietary recommendations for human populations.

Human experimental studies inevitably have greater applicability to human health than animal or cell models. Studies which use controlled dietary interventions are challenging to conduct, much more so than is the case for drug experimental trials where good adherence to the allocated intervention can be assured. Human studies involving individual foods or whole diets (as opposed to nutrients) also create additional challenges including how the intervention is delivered to optimise compliance (e.g. specialised foods, diet feeding studies, dietary advice studies) and against what the intervention should be compared, as placebo foods or placebo diets are complex.

The major advantage of animal or human dietary models is their potential to demonstrate a direct effect of the dietary intervention on a disease process or an intermediate marker for the disease. However, the detailed nature of some of the human studies, which can involve rigorous dietary interventions, specialised analytical and imaging techniques and biopsy sampling, which means they are rarely able to study large or representative groups of people.

The two approaches, whereby cohort studies reveal findings which reflect long-term dietary exposures from representative populations, and experimental studies which contribute biological plausibility, offer complementary lines of evidence to support the development of dietary recommendations. It is recognised that systematic processes which might optimise the selection of experimental findings in supporting the development of dietary recommendations are only in the early stages of development ${ }^{(19)}$. Further discussion on this topic can be found in the 'Standardisation and selection of evidence for biological plausibility' section.

\section{Processes and frameworks used to structure assessment of the evidence underpinning dietary recommendations}

Most authorities publish the frameworks and processes used by their expert groups to systematically assess, select and grade the evidence for adverse or beneficial effects of diet on noncommunicable diseases. The frameworks and processes used by four panels were examined: (i) WCRF Continuous Update Project $^{(14)}$, (ii) the Scientific Advisory Committee on Nutrition $(\mathrm{SACN})^{(20)}$, (iii) National Academies of Sciences, Engineering and Medicine and Dietary Guidelines Advisory Committee ${ }^{(21)}$ and (iv) the European Food Safety Authority ${ }^{(22)}$. The principles and methodologies used in assessing the overall strength of the evidence and for drawing conclusions and recommendations from a complex body of evidence are summarised under four main headings.

Systematic selection and assessment of the evidence. The diet-disease question(s) to be addressed and the types of studies to be included (e.g. RCTs, prospective cohort studies, casecontrol studies) are specified and agreed by the expert group. In most cases, an independent systematic review is undertaken, either by a specialist team or by the scientific staff of the organisations involved. These may be subject to external review or by a public call for evidence.

High-quality review protocols include pre-specification of the question(s) to be addressed; the search strategy (search engines, study type); search terms used; study relevance; study quality and statistical analysis of the summated data. 


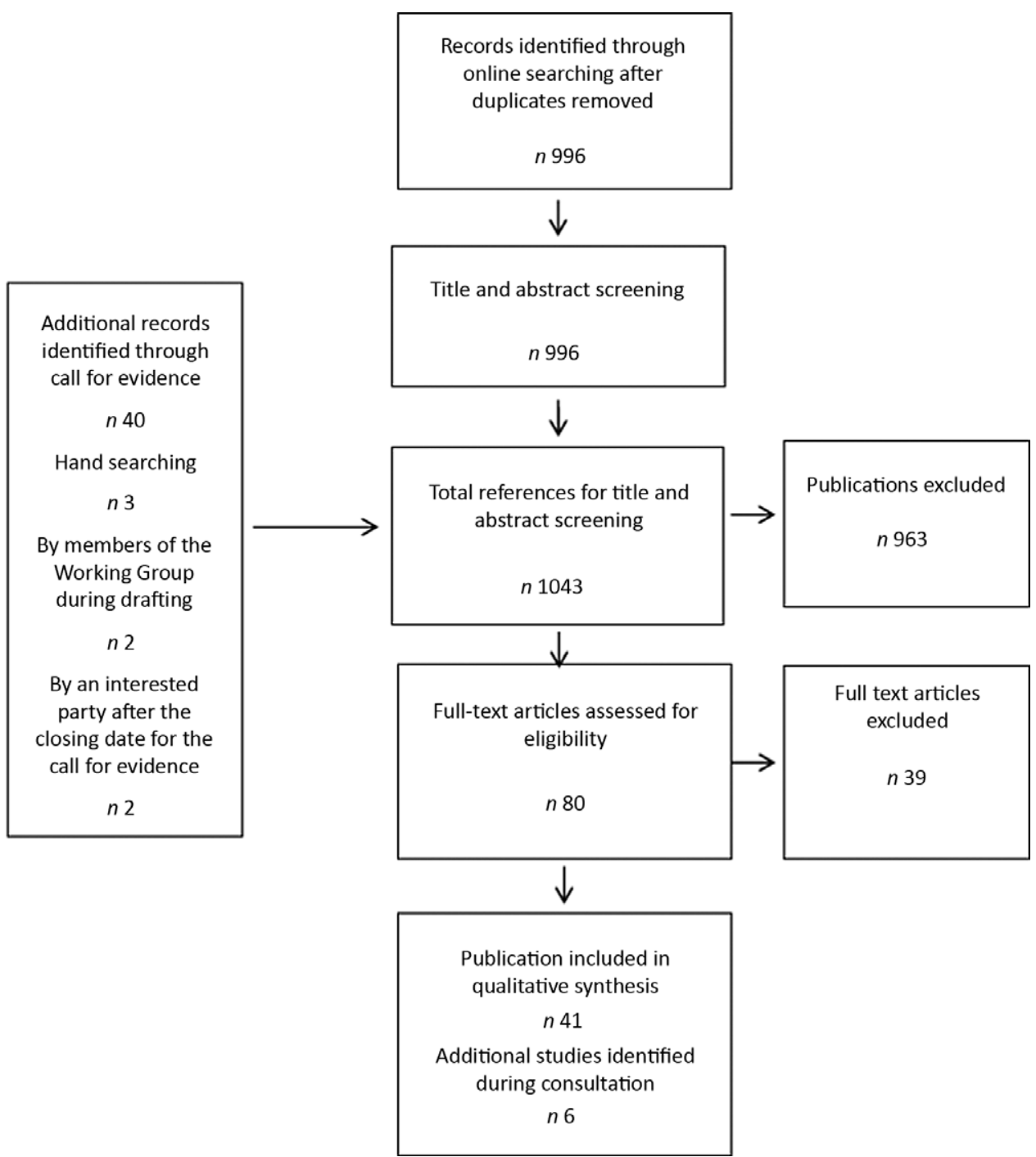

Fig. 1. Flow chart summarising the selection of published data used for the saturated fats and health: Scientific Advisory Committee on Nutrition report, 2019(23).

Quality criteria include rigour and relevance of the studies, including statistical approaches used, consideration of confounding, evidence for selection and publication bias and $a$ priori hypothesis testing.

As an example, Fig. 1 is a flow chart showing stages in selection of publications used in the SACN review of saturated fats and health, published in $2019^{(23)}$. Fig. 1 shows how most of the studies identified by the search terms to be relevant to the review were subsequently excluded by the review process for reasons such as lack of rigour, relevance or evidence of bias (including publication bias).

Systematic approaches to reviewing evidence for biological plausibility are not applied, as these have not yet been developed, although where evidence is considered relevant to the question being addressed and is consistent with the observational findings, they are considered for use by each of the four authorities.

Data are extracted from all studies used within the final report for subsequent tabulation and graphical summation of the findings.
Interrogation of the evidence for strength and adherence to causal characteristics. The outputs from the systematic reviews, including summated data in the form of meta- and pooled-analysis, are provided to the expert groups for consideration, with iteration between the expert and the scientific support groups to agree when further analysis is required. Interpretation of the evidence is guided through interrogation of the findings for adequacy of available data and adherence to causal characteristics including analysis of effect size; consistency of the findings from individual studies; dose-response relationships (including non-linear relationships); specificity; temporality and ability to eliminate confounding, error and bias (where possible).

Grading classification for overall evidence. Most expert panels use a grading system to define the level of certainty for causality in the evidence. These usually consist of four to five descriptors for levels of certainty for causal relationships (from high to low; Table 2). Judgements for making dietary recommendations are directly linked to the grading obtained from analysis 
Table 2. Examples of nomenclatures used to categorise the strength of the overall evidence for diet-disease relationships by four authorities

\begin{tabular}{|c|c|c|c|}
\hline EFSA $†$ & NASEM $\dagger$ & SACN & WCRF \\
\hline High* $^{*}$ & High $^{*}$ & Adequate* & Convincing - strong* \\
\hline Moderate* & Moderate* & Moderate* & Probable - strong* \\
\hline Low & Low & Limited & $\begin{array}{l}\text { Limited - suggestive } \\
\text { Limited - no conclusion }\end{array}$ \\
\hline Very low & Very low & $\begin{array}{l}\text { Inconsistent or } \\
\text { insufficient }\end{array}$ & $\begin{array}{l}\text { Substantial effect on risk } \\
\text { unlikely }\end{array}$ \\
\hline
\end{tabular}

EFSA, European Food Safety Authority; NASEM, National Academies of Sciences, Engineering, and Medicine; SACN, Scientific Advisory Committee on Nutrition; WCRF, World Cancer Research Fund.

* This level of evidence is considered sufficiently strong to allow dietary recommendations to be made.

† Use GRADE categories ${ }^{(15)}$.

of the evidence. Most authorities consider evidence is sufficient when graded within the top two categories of certainty according to their grading criteria.

Causal inference can be drawn where analysis of the overall evidence reveals effect sizes to be large and consistent, confounding and bias can be excluded and a clear dose-response is evident. Demonstration of temporality, where exposure can be confirmed to precede disease outcome, can be assessed from RCTs of diet-disease outcomes in humans, but few data of this type are available. Cohort studies also measure diet at baseline prior to disease outcome; subjects are excluded from analysis if they are diagnosed within a specified time from their baseline assessment, for example, 2 years.

In addition to findings from the systematic review analysis, the WCRF Continuous Update Project requires that to achieve the top two gradings in their framework (convincing and probable) the evidence must include assessment of biological mechanisms based on animal or human studies ${ }^{(14)}$. This requirement reflects the large amount of data available in the field of cancer biology, including mechanisms that have been shown to be influenced by diet and nutritional status. Other guidelines considered here do not define availability of evidence for biological plausibility as an a priori criteria for causality ${ }^{(20-22)}$, although experimental evidence is considered where available to provide biological context. No formal process for systematically selecting experimental data to be used in the panel's interrogation of this part of the evidence base is applied by any of the panels and relevant criteria are yet to be developed.

Making population dietary recommendations for prevention of non-communicable diseases. Although the guidelines and frameworks used by the four expert groups take broadly similar approaches to the selection, analysis and interrogation of the evidence (Fig. 2), there are some dissimilarities at this final stage in the process, where country-context is of particular importance. In the USA, the National Academies of Sciences, Engineering and Medicine develops and updates the guidance framework, while the evaluation of the evidence and development of dietary recommendations is undertaken by expert panels of the Dietary Guidelines Advisory Committee. In Europe, the European Food Safety Authority develops the framework and undertakes analysis of the evidence, but dietary recommendations are the responsibility of individual member state countries. In the UK, the assessment of the evidence (risk assessment) and identification of desirable dietary intakes are the responsibility of

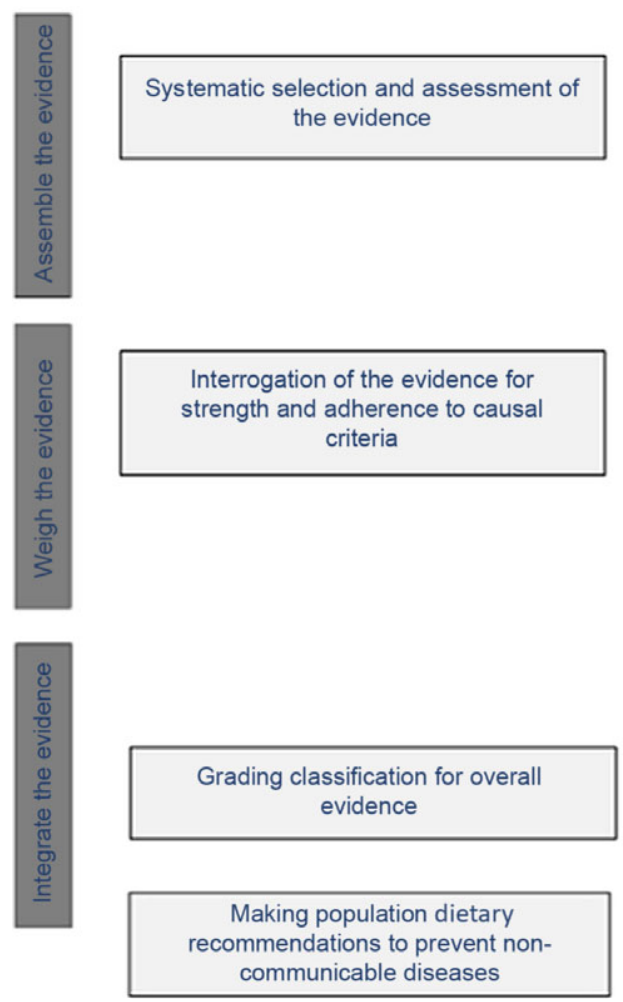

\begin{tabular}{|c|}
\hline $\begin{array}{l}\text { Diet-disease question(s) specified } \\
\text { Types of studies to be included and quality criteria agreed }\end{array}$ \\
\hline 52 \\
\hline Systematic review methods used to identify relevant information \\
\hline \\
\hline $\begin{array}{l}\text { Identify, filter and organise the evidence } \\
\text { Minimise confounding, error and selection bias } \\
\text { Data are extracted from studies; meta-analysis, tabulation and graphical } \\
\text { summation as required }\end{array}$ \\
\hline $\begin{array}{l}\text { Assessment of available data and adherence to causal criteria: } \\
\text { - } \quad \text { Analysis of effect size } \\
\text { - Consistency of the findings from individual studies } \\
\text { - Dose-response relationships } \\
\text { - Specificity } \\
\text { - Temporality } \\
\text { - Biological plausibility }\end{array}$ \\
\hline 5 \\
\hline $\begin{array}{l}\text { Assess relevance and reliability of the evidence } \\
\text { Assess consistency across the evidence } \\
\text { Levels of certainty for causal relationships agreed } \\
\text { Weight of evidence conclusion }\end{array}$ \\
\hline L \\
\hline $\begin{array}{l}\text { Assess and combine uncertainties and identify gaps } \\
\text { Conclusion of overall evidence }\end{array}$ \\
\hline
\end{tabular}

Fig. 2. Schematic representation of the processes used to structure assessment of the evidence underpinning dietary recommendations. 
the SACN; dietary recommendations and communication to the wider public (risk management) are the responsibility of government and policy makers. WCRF is an international cancer charity which funds research and, via the Continuous Update Project ${ }^{(14)}$, undertakes an ongoing evaluation of the evidence with the development of dietary recommendations at regular intervals. These cancers include a number of sites for which prevalence varies significantly between countries.

In making their final recommendations, expert panels will consider contextual factors beyond the quality and strength of the evidence. In particular, they will consider the prevalence of the non-communicable disease within the host country and the extent to which subject characteristics such as age distribution, sex, smoking and background diets of study cohorts differ from the population to which recommendations will apply. They will consider the feasibility of achieving the desired long-term changes in diet as well as cultural characteristics including religion, ethnicity and socio-economic factors, which may influence acceptability and compliance to population guidelines.

Aside from these empirical factors, different expert groups may not reach the same conclusions due to different judgements, perceptions and behaviours concerning the risks and benefits of particular dietary changes, despite similarity in the evidence base.

Country differences are not confined to decision-making concerning diet and health and indeed have been much in evidence during the recent global epidemic of Covid-19 where national infrastructures, health capacities and wider political considerations influenced the way in which countries responded to similar levels of infectious risk.

\section{Challenges in the evidence base}

\section{Standardisation and selection of evidence for biological} plausibility

Assessing the contribution that experimental studies can make to deriving population dietary recommendations is not straightforward (see the 'Processes and frameworks used to structure assessment of the evidence underpinning dietary recommendations' section). As well as concerns about their relevance to human dietary situations, the multiplicity and complexity of mechanisms which may underlie dietary or nutritional influences on chronic disease over time offer a significant interpretative challenge. Furthermore, efforts to systemise and summate findings from experimental studies have received much less attention than is the case for epidemiology. Agreed selection criteria for quality, relevance and applicability of various types of experimental studies, especially those related to human diet-disease outcomes, have not been fully established. This poses the risk that experimental data used by expert panels do not receive the same detailed scrutiny as those from epidemiology and limits the potential complementarities of the two approaches. A first step towards the development of a systematic review approach for experimental studies was reported in $2017^{(19)}$. This multi-disciplinary study developed a text mining protocol for objective identification and prioritisation of potential mechanisms for prostate cancer available in the search literature (total 37000 individual publications retrieved), with insulin-like-growth factor identified as a potential intermediate marker. This was followed by a modified systematic review to assess strength of the evidence for associations between dairy consumption, insulin-like-growth factor and prostate cancer. High heterogeneity in study design, methodology and treatment of results was found, which precluded use of conventional meta-analysis. Particular issues with cell studies included lack of basic quality control standards which resulted in exclusion of many studies due to lack of rigour. Evidence of positive publication bias and lack of duplication of mechanistic findings are additional concerns that have been raised by expert panels.

As well as adherence to rigorous methodology, where experimental models are to be used as part of the evidence base for dietary recommendations, it is imperative that criteria for relevance, including ability to apply the findings to human in vivo situations and use of feasible, nutritionally relevant dietary exposures, are assessed as part of the quality review. This will discriminate those studies whose findings can be used to support human dietary recommendations from those which cannot.

Further validation of systematic approaches to experimental findings, especially different approaches that might be used for cell, animal and human experimental models, is required. The development and acceptance of agreed criteria for systematic processes for this part of the literature will require coordinated, concerted effort, combined with training, peer review by international groups of experts, and sustained funding sources. Concerted effort from journal editors to require better reporting of studies and to include negative findings is key, as well as is ensuring appropriate peer review by funders to ensure the impact of their research investment is invested in appropriately designed studies. The conditions for supporting these developments require strategic prioritisation of resource that is currently lacking within the UK.

\section{Dietary assessment}

Developments in nutritional epidemiology and in the processes used to systemise the evaluation of their quality, relevance and consistency by expert committees have advanced significantly over the past 20 years. Methodological challenges remain, particularly with respect to the measurement of lifetime exposure to a variable as complex, changing and resistant to precise or accurate measurement as diet. A number of comprehensive reviews in this area have delineated the pros and cons of the various methodologies for estimating food and nutrient intakes. As with most scientific methods, dietary assessment is subject to measurement error, with effort to minimise this an active ongoing area of nutrition research methodology. Sources of error originate from inaccurate estimation of portion sizes; imperfect food composition tables; availability of a wide range of processed foods of varying and changing compositions; unrepresentative measurement periods and survey-related changes in behaviour resulting in selective, under- or misreporting of habitual diet ${ }^{(24)}$. Experienced investigators using standardised validated methods, selected according to the needs of the assessment, can reduce some sources of error. In recent years, digital 
recording of intakes and improved access to food compositional data, including through web-scraping of manufacturers' 'back of pack' nutrient composition data, as well as imaging of foods and meals, have enhanced the quality of record collection, eased the burden on participants and increased the feasibility of assessing diet within large-scale surveys ${ }^{(25)}$. Further research developments, such as identifying and validating biomarkers for specific food and nutrient intakes, including through metabolomic approaches, are ongoing ${ }^{(26)}$.

\section{Misclassification of diet, under-reporting and systematic bias}

FFQ provide the most feasible approach for assessing habitual diet in large-scale cohort studies ${ }^{(24)}$, although the method carries risk of misclassification of diet or food intakes. Most estimates for impact of dietary factors on disease risk are small (e.g. $20-30 \%$ increase or decrease in relative risk (RR)), so high heterogeneity in the data due to misclassification can obscure a true diet-effect relationship. Use of meta-analysis has provided an important advance since greater statistical power may enable detection of small effects of diet, although will not mitigate any biases in the original studies. For those relationships where the diet effect is small to moderate but the disease is common (e.g. colon cancer), successful achievement of dietary change has the potential for a large effect on population health.

A greater risk arises from the high prevalence of underreporting in dietary surveys. These arise from a number of sources including omission of specific foods from the record; under-estimation of portion sizes; under-reporting the frequency of eating or snacking; unrepresentative periods of recording; participant choice of food atypical of their habitual diets. Use of the doubly labelled water method for estimating energy expenditure from the UK National Diet and Nutrition Survey suggests reported energy intake in adults is more than $30 \%$ lower than actual intake estimated from energy expenditure measurements ${ }^{(27)}$. Many estimates of food intake now 'correct' for underreporting of energy intake, but these correction factors are only approximations and cannot account for omission of specific foods or beverages ${ }^{(28)}$. There are clear adverse implications for the validity of estimates of food, micronutrient and macronutrient composition derived from these correction factors.

Under-reporting will contribute to inaccuracy in estimates of food and nutrient intakes, and if not occurring equally across the population, may introduce systematic bias. Evidence from US and European populations suggests that under-reporting of energy intake is more prevalent in overweight and obese individuals especially women ${ }^{(29)}$. However, there may be crosscountry differences in obesity-related under-reporting since this was shown to be less prevalent in Egyptian than US women ${ }^{(30)}$. There is evidence that under-reporting has increased in prevalence and size over the past 20 years and is selective for certain high-energy macronutrients such as fat, sugar and alcohol ${ }^{(29)}$. Obesity-related under-reporting has the potential for systematic bias which becomes problematic when, for example, the analysis concerns relationships between an under-reported food or nutrient (e.g. fat or sugar) and specific diseases, such as CVD or cancers. It is notable that greater selective under-reporting of energy intake by overweight and obese subjects ( $>60 \%$ of UK adults) may explain the paradoxical observation of inverse relationships between dietary intakes of sucrose and body weight that have been reported from some dietary surveys ${ }^{(31)}$.

\section{Population changes in diet over time - interpretation of evidence from cohort studies}

Perhaps the greatest challenge for cohort studies of diet and noncommunicable diseases lies in the degree to which a single (or in some cases repeat) measurement describes an individual's diet over a period of 20-50 years. Many changes in people's diets will have taken place over this time period, with potential for widespread impact when these occur at population level. Changes of this type have occurred over the past 20-50 years, including the reduction in fat intakes in the UK between 1975 and $1995^{(32)}$, where intakes of saturated and monounsaturated fats fell by 32 and $29 \%$, respectively (Fig. 3(a)) due to reductions in total amount of fat consumed as well as differences in sources of fat (Fig. 3(b)). These changes largely occurred because new products developed in response to dietary guidelines were rapidly taken up by populations in the UK. Clearly, the exposure of interest (e.g. saturated fat) has changed and estimates derived from population cohorts after the changes in diet took place will not be the same as those estimated from cohorts studied prior to these changes. Risk to credibility of the findings is greatest for cohort studies that do not undertake repeat dietary measurement during the period of follow-up which was often the case with early studies.

An example of where a change in exposure has occurred comes from the Nurses' Health Study, where a strong association between trans-fats intakes and risk of CVD was first reported in 1993 ( $R R=1 \cdot 78$ ), with further reports from this cohort in 1997 and 2005, as reviewed by SACN in $2007^{(33)}$. Reputable manufacturers, concerned at the unexpected adverse findings from the 1993 report, began to modify their processes to remove trans-fats from margarines, and consumers began shifting their choices of margarines and oils at purchase (Fig. 3b). Risk estimates for trans-fats intakes and heart disease in this cohort were observed to reduce significantly between $1993(\mathrm{RR}=1.78), 1997(\mathrm{RR}=1.53)$ and 2005 ( $R R=1.33)$ as intakes of trans-fats fell within the US population. These dietary changes have continued through to the early 2000s, with trans-fats intakes in the UK declining from mean values of $>4 \%$ total energy in 1993 to values $<0.5 \%$ in current surveys ${ }^{(23)}$. Intakes at dietary levels $<2 \%$ have not been observed to have adverse effects on CVD risk or on markers of risk such as LDL-cholesterol and HDL-cholesterol concentrations

Implication of these changes in background diet are that unless accounted for in repeat diet assessments, these will lead to misleading risk estimates ongoing cohort studies. Risk estimates in the Nurses' Health Study were observed accurately because the present study measured diet every 4 years. Many cohorts do not undertake repeat diet measures, so baseline exposures are not representative of the subjects' diets over time. Additionally, new cohort studies established after a significant 
(a) 60

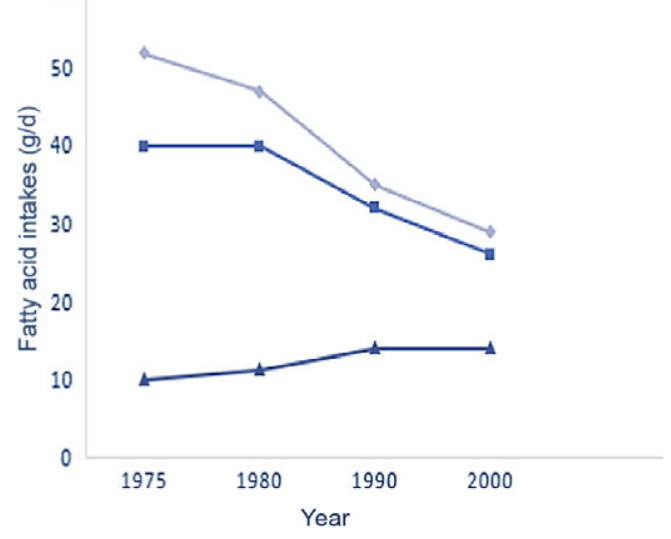

(b)

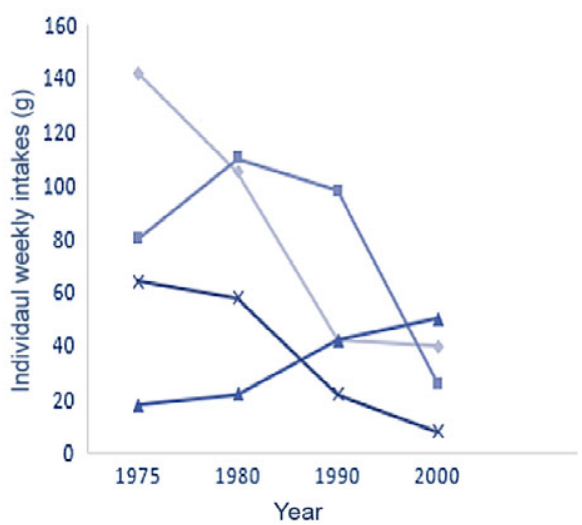

Fig. 3. Trends in intakes of fatty acids (a) and major fats and oils (b) in the UK, 1975-2000. Data from the Family Food Dataset (https://www.gov.uk/government/

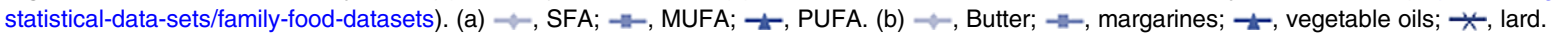

change in a population's diet will have quantitatively different intakes than cohorts studied earlier, contributing to greater heterogeneity and greater possibility of false null outcomes in meta-analyses.

There is a need for greater recognition of these issues since conflicting findings from recent $v$. earlier studies are taken as evidence by some commentators that the original findings must have been erroneous. In fact, if the original findings were correct, and the diet-disease association was causally related, then diminution of risk following changes in population diet would be the expected outcome. Factors such as these may have contributed to media stories that emerged in 2017 concerning saturated fat and CVD but were rarely raised in the debate. It should be recognised that long-term RCTs are likely to be similarly affected when controlled studies attempt to evaluate effects of a specific dietary change during a period when population diets are altering in the direction of the recommended intakes.

Changes in background diet over time are not the only confounding factors that can change over the course of long-term cohort studies but include also changes in body weight and fat, levels of physical activity, changes in smoking habits and prescribed drugs. While statistical models can be created, appropriate correction relies on understanding how these factors interact with one another pathogenically and whether these interactions are additive, synergistic or converse and how they interact over the life-course.

Considerations such as these illustrate why recommendations are made with great care, tend to err on the side of caution and remain open to re-evaluation with the possibility of change as greater and stronger evidence becomes available. They also reinforce the need for individual countries to undertake regular rolling assessment of dietary intakes and nutritional status against which findings from global cohort studies can be examined for their relevance and potential impact on their own populations' health.

\section{National surveillance of diet and nutritional status}

The National Health and Nutrition Examination Survey and the National Diet and Nutrition Survey programmes represent examples of long-standing national surveys of diet and nutrition.
These rolling programmes assess the diet and nutritional status of adults and children in the USA and the UK, respectively. They allow changes in food and nutrient intakes and nutritional status of populations to be monitored. They provide essential independent measures of temporal changes in diet and nutritional status, which can be taken into account when interpreting findings from long-term cohort studies, most of which rely on data from countries other than the UK. Through these surveys, national governments are able to assess the uptake of population advice on diet, as well as beneficial and potentially adverse consequences of any dietary change.

Unexpected and undesirable changes in nutrient intakes and nutritional status of vulnerable groups, such as infants, children and pregnant women, can be detected and acted upon where required. Increasing recognition of the impacts of inequality and ethnic diversity on aspects of diet, nutritional status and body fatness, which are strongly linked with non-communicable diseases, illustrates the potential value of UK nutritional surveillance data.

A contemporary example of the potential importance of national surveillance programmes is the recent focus on the environmental and health benefits of plant-based diets. Concerns for adverse environmental and health impacts of current food production systems and ability of plant-based diets to reduce these ${ }^{(34)}$ have been widely reported. Further research is needed to substantiate the potential benefits to the health of populations from the plant-based diets, as these are not yet perfectly understood nor universally accepted ${ }^{(35)}$. Nevertheless efforts being made to develop a wider range of plant-based foods indicate high likelihood of their widespread uptake over the next few years. This represents a typical example of a large-scale shift in eating habits that has high probability for confounding ongoing studies investigating benefits of plant-based diets as well as introducing significant changes in food and nutrient compositions of typical diets.

Replacing all or some animal foods with plant-based foods has been widely predicted to result in beneficial effects on health arising from known differences in the nutrient compositions of plant and animal products and reported health benefits linked with consumption of plant-based diets ${ }^{(34)}$. However, in the 
Table 3. Recommendations for dietary fat intakes for prevention of CVD in the UK from Committee on Medical Aspects of Food and Nutrition Policy (COMA) and Scientific Advisory Committee on Nutrition (SACN) reports 1974-2019

context of large-scale urbanised food production systems such effects are less predictable. Factors such as the impacts of existing and new forms of ingredients to add flavour and texture to plant-based ingredients, effects of different processing and cooking methods on food structure, bioavailability and on the gut microbiota, and their consequences for health are as yet incompletely understood. Assessment of this food transition will require monitoring of dietary intakes of the population for unintended consequences, including intakes of protein and micronutrients in vulnerable groups and of other nutrients such as saturated fats arising from fat substitution (e.g. from coconut oils) or salt for flavour enhancement. Periods of increased need such as infancy, childhood, adolescence, pregnancy and lactation, when optimal absorption and uptake of nutrients are of key importance, will require particular focus. National surveillance data will be central to these assessments because of their ability to assess impact in particular sub-groups of the population, where small ad hoc studies will be insufficient to inform future public health policy.

\section{Communication about findings from nutrition research}

Media stories on nutrition often assert that the evidence keeps changing, current dietary approaches to health are incorrect or that findings from a new study have overthrown existing dogma. This paper recognises the potential for confusion and has examined evidence that might support arguments that formal recommendations for dietary changes have been frequent and/or inconsistent. While the evidence base for nutrition is undoubtedly an incremental one, the review has not found large numbers of examples where formal recommendations from government or other recommending authorities have changed frequently. Below are examples where recommendations have remained consistent over long periods of repeated updating of the evidence, as well as others where evidence has become available from stronger studies that are not in line with the original findings, leading to a change in recommendation.

\section{Examples where dietary recommendations have stayed consistent following updating with newly available evidence}

Fats and CVD. Media articles often claim the evidence for saturated fats has changed due to new or different analyses of existing evidence. Table 3 summarises 45 years coverage of this evidence via UK expert reviews of diet and CVD risk from 1974 to 2019. The recommendation that saturated fats should be reduced from the high levels consumed in 1974 (17\% dietary energy $)^{(8)}$ has been a consistent outcome from each of the reports that have examined this evidence. In 1984, the recommendation was made that the combined total of saturated and trans-fats intakes should be no more than $15 \%$ energy (at the time trans-fats intakes were $4.6 \%$ of energy intakes) ${ }^{(36)}$. Since 1994, the UK recommendation has been that saturated fats should comprise no more than $10 \%$, and trans-fats less than $2 \%$ of dietary energy intakes ${ }^{(37)}$, a position that remains unchanged in the 2019 report $^{(23)}$. The major change for the 2019 recommendations is that saturated fat intakes should be reduced below $10 \%$ energy by replacement with unsaturated fats, while polyunsaturated fats should not exceed $10 \%$ dietary energy.

Diet and cancer. Examples of consistent recommendations for diet and nutritional risk factors for certain cancers are shown in Table 4. These data summarise the gradings for strength of the evidence for diet and nutritional status with respect to colorectal cancer for the period 1997-2018, with one report from COMA ${ }^{(40)}$ and three reports from $\mathrm{WCRF}^{(41-43)}$. Over a period of 20 years, during which there has been marked expansion in the volume of evidence, there have been few changes in the main findings. In the WCRF framework, evidence graded convincing or probable is considered sufficiently strong to justify making dietary recommendations for increasing or reducing intakes of specific foods. Findings of convincing or probable evidence were demonstrated for increased risk of colorectal cancer with red and processed meat, alcoholic drinks, body fatness, abdominal fat and attained adult height. Findings at probable levels of evidence 
Table 4. Grading of the strength of the evidence for relationship between specific dietary components or anthropometric measures and risk of colorectal cancer, Committee on Medical Aspects of Food and Nutrition Policy (COMA)*; World Cancer Research Fund (WCRF) $\dagger$

\begin{tabular}{|c|c|c|c|c|}
\hline & COMA $1998^{(40)}$ & WCRF $1997^{(41)}$ & WCRF $2007^{(42)}$ & WCRF $2018^{(43)}$ \\
\hline \multicolumn{5}{|c|}{ Decreased risk } \\
\hline Vegetables & Moderate & Limited - suggestive & Limited - suggestive & Limited - suggestive \\
\hline Whole grain & - & - & - & Probable \\
\hline Foods containing fibre & Moderate & Probable & Convincing & Probable \\
\hline Garlic & - & Probable & Probable & Inconsistent - no conclusions drawn \\
\hline Milk & - & Probable & Probable & Probable \\
\hline Dairy & - & - & - & \\
\hline Calcium & - & $\begin{array}{l}\text { Probable } \\
\quad \text { Increased risk }\end{array}$ & Probable & Probable§ \\
\hline Red meat & Moderate (red and processed & Convincing & Convincing & Probablell \\
\hline Processed meat & meat together) & Convincing & Convincing & Convincing \\
\hline Alcoholic drinks & - & Convincing (men) & Convincing (men) & Convincing \\
\hline & & Probable (women) & Probable (women) & (men and women) \\
\hline Body fatness & Weak & Convincing & Convincing & Convincing \\
\hline Abdominal fatness & - & Convincing & Convincing & Convincing \\
\hline Adult attained height & - & Convincing & Convincing & Convincing \\
\hline
\end{tabular}

* Strength classified as: strong; moderate; weak; not enough.

† Strength classified as: convincing; probable; limited - suggestive; limited - no conclusion; substantial effect on risk unlikely.

¥ Includes dairy, milk, cheese and dietary $\mathrm{Ca}$.

$\S$ Includes Ca supplements $>200 \mathrm{mg} / \mathrm{d}$.

II Includes beef, lamb, pork and goat.

for protective effects against colorectal cancer were reported for foods containing fibre, whole grain, dairy, milk and Ca.

The strength and consistency of the evidence for links between body fatness and abdominal fatness and risk of some cancers is a notable feature of the cancer evidence base, with ratings of convincing for a number of sites including colorectal, breast, pancreas and liver. These assessments are based on not only the consistency of the epidemiology but also evidence for plausible biological mechanisms which can explain the impact of adiposity on cancer pathophysiology. The strength of these data may be explained in part by the relative ease and consistency of reporting for anthropometric measures such as weight and adult weight gain, height, waist and hip circumference, compared with diet. As biomarkers of energy balance throughout adult life, measures such as BMI for total fatness and waist circumference for abdominal fatness, as well as weight gain during adult life provide strong evidence of the importance of maintaining a constant adult body weight through diet and physical activity on risk of certain cancers.

Examples where dietary recommendations have not stayed consistent following updating with newly available evidence

Where more evidence, or evidence of greater quality, becomes available, this is considered within the context of the existing evidence base, with changes to dietary recommendations made when the grading of the evidence moves above or below the category for making dietary recommendations.

An example of where the WCRF recommendations have altered because the evidence base has changed is for fruit and non-starchy vegetables and several cancers. The 1997 WCRF report gave a strong recommendation for a protective effect at a number of cancer sites, based mainly on case-control studies. However, by 2007 and in 2018, the conclusion had changed to limited-suggestive because the epidemiological evidence had advanced through the inclusion of more cohort studies, which did not support the case-control evidence. This category change from strong to limited-suggestive also led to a change in the dietary recommendations. A similar change was made in the 2018 recommendations for reducing salt intakes for prevention of stomach cancer. Although evidence was rated as strong in the 2007 report, this was changed to limited-suggestive in 2018 due to evidence from cohort studies that did not support the earlier evidence.

The 2017 SACN report on carbohydrates and health ${ }^{(44)}$ made recommendations for increased intakes of dietary fibre (from 24 to $30 \mathrm{~g} / \mathrm{d}$ ) due to stronger evidence for protection against cardiometabolic disease and colorectal cancer. This report also recommended that sugars intakes should not exceed 5\% dietary energy due to evidence that increasing sugars intake leads to higher energy intakes. These recommendations were made against increasing concern for the high rates of obesity and overweight in UK adults and of rising incidence in children.

\section{Future communications}

Many of the problems of miscommunication in diet and health arise from findings which are reported out of context and not against the background of the totality of the evidence. Diet and health are popular topics for debate in scientific and nonscientific media, and individual scientists are frequently asked to comment on new studies that the media wish to highlight because they appear to conflict with current dietary advice. Some of these are evidently well-conducted large-scale studies which are likely to make a contribution to the totality of the evidence base and will be considered as part of the evidence if they fulfil the relevant criteria from a systematic review. Others include new experimental findings about possible dietary-mediated mechanisms that can support findings from observational studies and represent important and novel findings. Nevertheless, there are also reports that receive inappropriate attention because they appear to make unsubstantiated claims about their importance or 
originate from less substantial studies that may not be rigorously designed, or lack relevance to human diet and health, and which require confirmation via larger or stronger studies.

There has been increasing emphasis on the need for researchers to demonstrate the impact of their research including via reports of their findings in the media and in non-scientific publications. These developments have been supported by the UK Research Excellence Framework and have played an important part in recognising the considerable benefits which research brings to economic and human well-being. Many universities and research institutes have media and communication professionals who are adept at identifying attractive stories from their institutions with, understandably, diet and health representing a key topic for attention. However, there is also concern that these developments have contributed to greater pressure on researchers, especially early career nutrition researchers who need to present their work at meetings in order to gain experience. Finding a balanced approach to this will be needed since early career researchers benefit from the experience of developing their work for publication, including responding to critiques from their peers in the same way as other scientists.

There are a number of organisations whose work helps to support a consistent focus on evidence-based nutrition findings ${ }^{(45,46)}$ and many individual scientists, senior as well as early career, contribute time to communicating complex areas of nutrition and health in the media. The Academy recognises the need to ensure more effective translation of nutrition science in the media and to lay audiences, as well as within specialist groups such as health professionals and other scientists. There is a shared responsibility between researchers, funders and policy makers to support better understanding of the nature of the evidence base in order to strengthen the credibility of the science and provide confidence in public health policies based upon it.

\section{Conclusions and recommendations}

This position paper focuses explicitly on the evidence which underpins dietary recommendations for prevention of noncommunicable diseases. It considers the developments made in systematising the epidemiological evidence and the frameworks used by expert groups to achieve objective, rigorous and transparent outcomes from their work. Notably, the Academy did not find major differences in the approaches and processes used by expert panels across the UK, mainland Europe or the USA nor by the WCRF Continuous Update Project process.

The open nature of the work and the systematic methods, processes and criteria used by them to achieve dietary recommendations for population health were considered to be as rigorous as in other areas of science, medicine and health ${ }^{(47)}$. Further, frameworks that allow regular updates from published findings were recognised as important developments.

However, some challenges remain. The dependence of the evidence base on observational research has represented a challenge for expert groups since these were first set up in the 1970s and 1980 s due to the difficulty in ascribing causality from observed associations. Advances in methodologies and statistical approaches, alongside a greater number of well-powered cohort studies, have improved this position to some extent. However, a remaining challenge is that RCTs, normally considered the 'gold standard' for demonstrating causality in clinical medicine (where clinical outcomes are the primary end-point), have proved to be far less useful in studying diet-disease relationships for several reasons including the cost and complexity of assuring long-term dietary compliance in large numbers of subjects $^{(3)}$.

The Academy considers that experimental studies which investigate biological mechanisms underlying diet-disease relationships have not been sufficiently exploited for assessing diet and health relationships. Observational and experimental findings contribute complementary information that can strengthen the evidence for causal relationships. A more systematic approach to this part of the evidence base, including clear criteria for selection of studies based on quality, rigour and relevance, should enable discrimination of those studies that can usefully address current diet and health questions from those that cannot.

Diet has long been acknowledged as a challenging exposure for epidemiological investigation because the potential for confounding and bias is greater for diet than for many other exposures. The Academy considers these challenges have increased substantially since the latter part of the twentieth century. These include the variety and frequency of compositional changes in manufactured foods; confounding due to changes in population diets as well as other environmental exposures (physical activity, smoking, drugs) over time and high prevalence and extent of under-reporting of energy intakes.

Greater recognition needs to be given to the significance of changes in habitual background diets including those arising from the uptake of population dietary recommendations since these have potential to confound findings from ongoing cohort studies. As well as the need to address these and changes in other possible confounding exposures within the published literature, the Academy considers national nutrition surveillance programmes provide essential independent measures of temporal changes in diet and nutritional status since these can be taken into account by expert panels when interpreting the relevance and priority of findings from studies conducted outside the host country.

The Academy has examined evidence that might support arguments that recommendations for diets are frequently changing or inconsistent. The possibility that confusion could undermine attempts to achieve desirable population changes in diet is a serious consideration for the Academy. For two of the main diseases for which diet has been causally implicated (CVD and colon cancer), the Academy found that the main dietary recommendations for reducing disease risk had not substantially changed over time. The recommendations for saturated fat and CVD have been consistent for 50 years and those for diet, body weight and colon cancer for at least 20 years. Where changes have been made, these have been due to changes in the evidence base due to availability of new data from highquality cohort studies and lack of a clear mechanism to support observational findings. The Academy considered that reporting 
of individual research findings in the media may have contributed to the perception of frequent changes in dietary recommendations. This position paper notes and supports the key principle of evaluating the totality of available evidence through selection and interrogation according to rigour and relevance. In this regard, it is noted that some reports in the media, including the scientific media, assert a degree of certainty and level of significance for single findings that are not always warranted.

The Academy considered the nutrition profession as a whole could do more to communicate the rigorous processes involved in making dietary recommendations. While expert reports are widely available and their processes open to transparency and scrutiny, the detailed and specialist nature of their content is not easily accessible to non-specialists including media professionals. Audience-specific communications should be developed, including different categories of health professionals, media, other scientists and lay audiences. There is also need for researchers and media professionals to work together, at an institutional level, to achieve a balanced approach to demonstrating the impact of their research.

The Trustees of the Academy have agreed to set up a Communications Working Group to consider how nutrition professionals can work with various stakeholders to improve greater visibility and understanding of the evidence underpinning recommendations for diet and health.

\section{Recommendations}

The Academy makes a number of recommendations relating to its main conclusions:

1. Better translation of experimental findings for diet and disease. There should be further development of approaches that allow the systematic selection of experimental, biological evidence according to criteria for quality and nutritional relevance. This body of evidence needs to be used in an objective, non-biased and transparent way in assessing relationships between diet and disease.

This recommendation is relevant for a number of Academy stakeholders including government bodies making recommendations on diet and health, funding agencies and journal editors undertaking peer review of experimental research in nutrition. Researchers and research funding agencies are important stakeholders, including those from cognate science disciplines whose work is concerned with investigating how diets, foods or nutrients influence the pathogenesis of human disease. The conditions for supporting these developments require strategic prioritisation of resource that is currently lacking within the UK.

2. Novel methodologies in dietary assessment and nutritional epidemiology. There is a continuing need to develop and share innovative ways of measuring dietary exposures, reducing misreporting and correcting for confounding effects of changes in background diets within existing and new cohort studies.
This recommendation is relevant to the Academy of Nutrition Sciences member science organisations, research funding agencies and journal editors, to ensure dissemination and translation of best practice and avoid duplication of effort.

3. The rolling national nutrition surveillance programmes should continue. They provide independent measures of temporal trends in diet and nutritional status that are required to interpret the significance, relevance and priority of findings from global cohort studies that may impact on UK population health.

The government body responsible for this national infrastructure is presently Public Health England, but this may change as this organisation undergoes transition to the new structures. The Academy commends the national nutrition surveillance programme as a key infrastructure for prioritising work on all aspects of public health nutrition including that related to both infectious and non-communicable disease.

\section{Acknowledgements}

The authors acknowledge the helpful comments made on drafts of this review by members of the Board of Trustees of the Academy of Nutrition Sciences, as well as independent comments from Professors Janet Cade, Hillary Powers and Martin Wiseman.

C. M. W. initiated the review and drafted the early version for discussion; Co-authors (M. A., M. H., A. P. and S. S.) contributed equally to the discussion and development of the early content into the present report including through: critique of the early content; writing and reviewing of additional sections; preparation of graphs and tables and reading and approving the final version.

There are no conflicts of interest.

\section{References}

1. Gibney M, Allison D, Bier D, et al. (2020) Uncertainty in human nutrition research. Nat Food 1, 247-249.

2. Schulze MB, Martínez-González MA, Fung TT, et al. (2018) Food based dietary patterns and chronic disease prevention. BMJ 361, k2396.

3. Giovannucci E (2018) Nutritional epidemiology and cancer: a tale of two cities. Cancer Causes Control 29, 1007-1014.

4. University of Minnesota (2020) A History of Cardiovascular Disease Epidemiology. Early Era: 1940s-1970s. http://www. epi.umn.edu/crdepi/history-era/early-era-1940s-1970s/ (accessed July 2020).

5. National Research Council (US) Committee on Diet Nutrition and Cancer (1982) Diet, Nutrition, and Cancer. Washington, DC: National Academies Press (US).

6. World Health Organization (1982) Prevention of Coronary Heart Disease: Report of a WHO Expert Committee. Technical report series: no. 678. Geneva: World Health Organization.

7. American Heart Association (1982) Rationale of the diet-heart statement of the American Heart Association. Report of the AHA Nutrition Committee. Arteriosclerosis 2, 177-191. 
8. DHSS (Department of Health and Social Security) (1974) Diet and Coronary Heart Disease. Report of the Advisory Panel of the Committee on Medical Aspects of Food Policy (Nutrition) on Diet in Relation to Cardiovascular and Cerebrovascular Disease. https://www.gov.uk/government/publications/comareports (accessed July 2020).

9. Bradford-Hill A (1965) The environment and disease: association or causation? Proc R Soc Med 58, 295-300.

10. Lichtenstein AH, Yetley EA \& Lau J (2008) Application of systematic review methodology to the field of nutrition. $J$ Nutr 138, 2297-2306.

11. Higgins JPT, Thomas J, Chandler J, et al. (editors) (2019) Cochrane Handbook for Systematic Reviews of Interventions version 6.0 (updated July 2019). www.training.cochrane.org/ handbook (accessed July 2020).

12. World Cancer Research Fund (2018) Systematic Literature Review Specification Manual. http://www.dietandcancerreport.org/?p= slr_specification_manual (accessed July 2020).

13. University of York Centre for Review and Dissemination (2008) Systematic Reviews: CRD's Guidance for Undertaking Reviews in Health Care. https://www.york.ac.uk/crd/guidance/ (accessed July 2020).

14. World Cancer Research Fund/American Institute for Cancer Research (2018) Continuous Update Project Update Report: Judging the Evidence. https://www.wcrf.org/dietandcancer/ judging-evidence (accessed July 2020)

15. Guyatt GH, Oxman AD, Vist GE, et al. (2008) GRADE: an emerging consensus on rating quality of evidence and strength of recommendations. BMJ 336, 924-926.

16. Shea BJ, Reeves BC, Wells G, et al. (2017) AMSTAR 2: a critical appraisal tool for systematic reviews that include randomised or non-randomised studies of healthcare interventions, or both. BMJ 358, j4008.

17. Katz D, Karlsen M, Chung M, et al. (2019) Hierarchies of Evidence Applied to Lifestyle Medicine (HEALM): introduction of a strength-of-evidence approach based on a methodological systematic review. BMC Med Res Meth 19, 178

18. Schwingshackl L, Knüppel S, Schwedhelm C, et al. (2016) Perspective: NutriGrade: a scoring system to assess and judge the meta-evidence of randomized controlled trials and cohort studies in nutrition research. Adv Nutr 7, 994-1004.

19. Lewis SJ, Gardner M, Higgins J, et al. (2017) Developing the WCRF International/University of Bristol methodology for identifying and carrying out systematic reviews of mechanisms of exposure-cancer associations. Cancer Epidemiol Biomarker Prev 26, 1667-1675.

20. Scientific Advisory Committee on Nutrition (2020) A Framework for Evaluation of Evidence that Relates Food and Nutrients to Health (updated March 2020). https://www.gov. uk/government/groups/scientific-advisory-committee-onnutrition\#framework-for-the-evaluation-of-evidence (accessed July 2020).

21. NASEM (National Academies of Sciences Engineering and Medicine) (2017) Guiding Principles for Developing Dietary Reference Intakes Based on Chronic Disease. https://doi.org/ 10.17226/24828 (accessed July 2020).

22. EFSA Scientific Committee (2017) Scientific opinion on the guidance on the use of the weight of evidence approach in scientific assessments. EFSA J 15, 4971.

23. Scientific Advisory Committee on Nutrition (2020) Saturated Fats and Health: SACN Report. Public Health England. www. gov.uk/government/publications/saturated-fats-and-healthsacn-report (accessed July 2020).

24. Thompson FE, Kirkpatrick SL, Subar AF, et al. (2015) The National Cancer Institute's dietary assessment primer: a resource for diet research. J Acad Nutr Diet 66, 1986-1995.
25. Cade J (2017) Measuring diet in the 21st century: use of new technologies. Proc Nutr Soc 76, 276-282.

26. Hedrick VE, Dietrich AM, Estabrooks PA, et al. (2012) Dietary biomarkers: advances, limitations and future directions. Nutr J 11, 109.

27. Bates B, Lennox A, Prentice A, et al. (2014) National Diet and Nutrition Survey results from years 1,2,3 and 4 (combined) of the rolling programme (2008/2009-2011/2012) (revised February 2017). https://www.gov.uk/government/statistics/ national-diet-and-nutrition-survey-results-from-years-1-to4-combined-of-the-rolling-programme-for-2008-and-2009to-2011-and-2012 (accessed July 2020).

28. Tooze JA, Krebs-Smith SM, Troiano RP, et al. (2012) The accuracy of the Goldberg method for classifying misreporters of energy intake on a food frequency questionnaire and 24-h recalls: comparison with doubly labeled water. Eur J Clin Nur 66, 569-576.

29. Macdiarmid J \& Blundell J (1998) Assessing dietary intake: who, what and why of under-reporting. Nutr Res Rev 11, 231-253.

30. Harrison GG, Galal OM, Ibrahim N, et al. (2000) Underreporting of food intake by dietary recall is not universal: a comparison of data from Egyptian and American women. J Nutr 130, 2049-2054.

31. Kuhnle GG, Tasevska N, Lentjes MA, et al. (2015) Association between sucrose intake and risk of overweight and obesity in a prospective sub-cohort of the European Prospective Investigation into Cancer in Norfolk (EPIC-Norfolk). Public Health Nutr 18, 2815-2824.

32. Foster R \& Lunn J (2007) 40th Anniversary briefing paper: food availability and our changing diet. Nutr Bull 32, 187-249.

33. Scientific Advisory Committee on Nutrition (2007) Update on trans fatty acids and health. Position statement by the Scientific Advisory Committee on Nutrition. https://www.gov.uk/ government/publications/sacn-update-on-trans-fatty-acids-2007 (accessed July 2020).

34. Willett W, Rockström J, Loken B, et al. (2019) Food in the anthropocene: the EAT-Lancet commission on healthy diets from sustainable food systems. Lancet 393, 447-492.

35. Zagmutt FJ, Pouzou JG \& Costard S (2020) The EAT-Lancet commission's dietary composition may not prevent non-communicable disease mortality. J Nutr 150, 985-988.

36. COMA (Committee on Medical Aspects of Food Policy) (1984) Diet and Cardiovascular Disease. Committee on Medical Aspects of Food Policy. Report of the panel on diet in relation to cardiovascular disease. Rep Health Soc Subj 28, 1-32.

37. COMA (Committee on Medical Aspects of Food Policy) (1994) Nutritional Aspects of Cardiovascular Disease. https://www. gov.uk/government/publications/coma-reports (accessed July 2020).

38. Scientific Advisory Committee on Nutrition and Committee on Toxicity (2004) Advice on fish consumption: benefits and risks. https://www.gov.uk/government/publications/sacnadvice-on-fish-consumption (accessed July 2020).

39. COMA (Committee on Medical Aspects of Food Policy) (1991) Dietary reference values for food energy and nutrients for the United Kingdom. Rep Health Soc Subj (Lond) 41, 1-210.

40. COMA (Committee on Medical Aspects of Food Policy) (1998) Nutritional aspects of the development of cancer. Report of the working group on diet and cancer of the committee on medical aspects of food and nutrition policy. Rep Health Soc Subj (Lond) 48, i-xiv, 1-274.

41. World Cancer Research Fund/American Institute for Cancer Research (1997) Diet, Nutrition, Physical Activity and Cancer: A Global Perspective. Continuous Update Project Expert Report. Washington, DC: American Institute for Cancer Research. 
42. World Cancer Research Fund/American Institute for Cancer Research (2007) Diet, Nutrition, Physical Activity and Cancer: A Global Perspective. Continuous Update Project Expert Report. Washington, DC: American Institute for Cancer Research.

43. World Cancer Research Fund/American Institute for Cancer Research (2018) Diet, Nutrition, Physical Activity and Cancer: a Global Perspective. Continuous Update Project Third Expert Report. www.dietandcancerreport.org (accessed July 2020).

44. Scientific Advisory Committee on Nutrition (2015) Carbohydrates and Health. https://assets.publishing.service.gov.uk/government/
uploads/system/uploads/attachment_data/file/445503/SACN Carbohydrates_and_Health.pdf (accessed August 2020).

45. The Science Media Centre (2020) The Science Media Centre home page. www.sciencemediacentre.org (accessed July 2020).

46. The British Nutrition Foundation (2020) The British Nutrition Foundation home page. www.nutrition.org.uk (accessed July 2020).

47. National Institute for Health and Care Excellence (2020) NICE guidance. https://www.nice.org.uk/guidance (accessed July 2020). 